\title{
Exact-exchange Kohn-Sham potential, surface energy, and work function of jellium slabs
}

\author{
C. M. Horowitz, ${ }^{1,2}$ C. R. Proetto, ${ }^{3, *}$ and J. M. Pitarke ${ }^{4,5}$ \\ ${ }^{1}$ Donostia International Physics Center (DIPC), E-20018 San Sebastian, Spain \\ ${ }^{2}$ Instituto de Investigaciones Fisicoquímicas Teóricas y Aplicadas, (INIFTA), UNLP, CCT La Plata-CONICET, Sucursal 4, \\ Casilla de Correo 16, (1900) La Plata, Argentina \\ ${ }^{3}$ Institüt für Teoretische Physik, Freie Universitat Berlin, Arnimallee 14, D-14195 Berlin, Germany \\ and European Theoretical Spectroscopy Facility (ETSF) \\ ${ }^{4}$ CIC nanoGUNE Consolider, Mikeletegi Pasealekua 56, E-20009 Donostia, Basque Country, Spain \\ ${ }^{5}$ Materia Kondentsatuaren Fisika Saila and Centro Física Materiales, CSIC-UPV/EHU, 644 Posta Kutxatila, E-48080 Bilbo, \\ Basque Country, Spain
}

(Received 22 May 2008; revised manuscript received 22 July 2008; published 25 August 2008)

\begin{abstract}
Exact-exchange self-consistent calculations of the Kohn-Sham potential, surface energy, and work function of jellium slabs are reported in the framework of the optimized effective potential (OEP) scheme of density functional theory. In the vacuum side of the jellium surface and at a distance $z$ that is larger than the slab thickness, the exchange-only Kohn-Sham potential is found to be imagelike $\left(\sim-e^{2} / z\right)$ but with a coefficient that differs from that of the classical image potential $V_{\mathrm{im}}(z)=-e^{2} / 4 z$. The three OEP contributions to the surface energy (kinetic, electrostatic, and exchange) are found to oscillate as a function of the slab thickness, as occurs in the case of the corresponding calculations based on the use of single-particle orbitals and energies obtained in the local-density approximation (LDA). The OEP work function presents large quantum size effects that are absent in the LDA and which reflect the intrinsic derivative discontinuity of the exact Kohn-Sham potential.
\end{abstract}

DOI: 10.1103/PhysRevB.78.085126

PACS number(s): 31.10.+z, 31.15.E-

\section{INTRODUCTION}

The analysis of the electronic structure of metal surfaces poses a big theoretical challenge; a suitable calculational tool is needed for large, interacting, and strongly inhomogeneous many-electron systems. More than 30 years since its first application by Lang and Kohn ${ }^{1,2}$ to the surface problem, little doubt exists that one method of choice for fulfilling this goal is density functional theory (DFT). ${ }^{3,4}$ DFT aims to a microscopic understanding of atoms, molecules, clusters, surfaces, and bulk solids starting from the fundamental laws of quantum mechanics. In the Kohn-Sham ${ }^{5}$ (KS) implementation of DFT, the complicated many-body problem is mapped to an effective single-particle problem, with particles subjected to an effective single-particle potential (the KS potential). Although this mapping is exact, it gives no clue as to how to calculate in practice the so-called exchange-correlation (xc) contribution to the KS potential. Lang and Kohn ${ }^{1,2}$ solved this problem by using the local-density approximation (LDA) for the surface problem. In LDA, the xc potential at each point is taken to be that of a homogeneous interacting electron gas with the local density. Since then, many authors have calculated the electronic properties of metal surfaces by using either the LDA (Ref. 6) or further elaborations that incorporate nonlocal ingredients to the unknown $\mathrm{xc}$ functional. ${ }^{7,8}$ Other schemes of the computational electronicstructure tool kit available for the investigation of solid surfaces are the Fermi hypernetted-chain (FHNC) method, ${ }^{9,10}$ the $G W$ approximation, ${ }^{11}$ quantum Monte Carlo (QMC), ${ }^{12-14}$ and the inhomogeneous Singwi-Tosi-Land-Sjölander (ISTLS) approach. ${ }^{15}$

In the framework of the optimized effective potential (OEP) scheme of DFT, ${ }^{16,17}$ which had been first used in the context of atomic physics, ${ }^{18}$ correlation is ignored altogether and the exact-exchange KS potential is obtained. Several advantages are associated with the use of the exact-exchange energy functional of DFT: (i) it corrects the self-interaction problem inherent in approximate treatments of the exchange energy $^{19}$ (this problem is particularly acute for localized systems such as atoms and molecules, although it is not relevant for extended systems like bulk solids and solid surfaces); (ii) it yields great improvements in the study of the KS eigenvalue spectrum, ${ }^{20}$ semiconductor band structures and excitations, ${ }^{21}$ and nonlinear optical properties; ${ }^{22}$ (iii) it yields the correct asymptotics; ${ }^{23}$ (iv) it reproduces the derivative discontinuity which should be present in the KS exchange potential each time the number of particles crosses through an integer value; ${ }^{24-28}$ and (v) it yields the correct twodimensional (2D) exchange energy per particle in the case of a quasi-2D electron gas. ${ }^{29}$ It is the aim of this paper to provide benchmark exact-exchange OEP calculations for jellium slabs, with the expectation that more accurate DFT schemes that include correlation be developed by starting from a well founded exchange analysis and tested once reduced to their exchange-only ( $x$-only) counterparts.

The rest of the paper is organized as follows. We give in Sec. II the general theoretical background which will be used in the following sections. Section III is devoted to a discussion of the asymptotic behavior of the exact-exchange KS potential of jellium slabs; in Secs. IV and V we give the results that we have obtained for the OEP surface energy and work-function, respectively, and in Sec. VI we present the conclusions.

\section{OEP APPROACH}

Our calculations are restricted to a jellium-slab model of metal surfaces, where the discrete character of the positive 

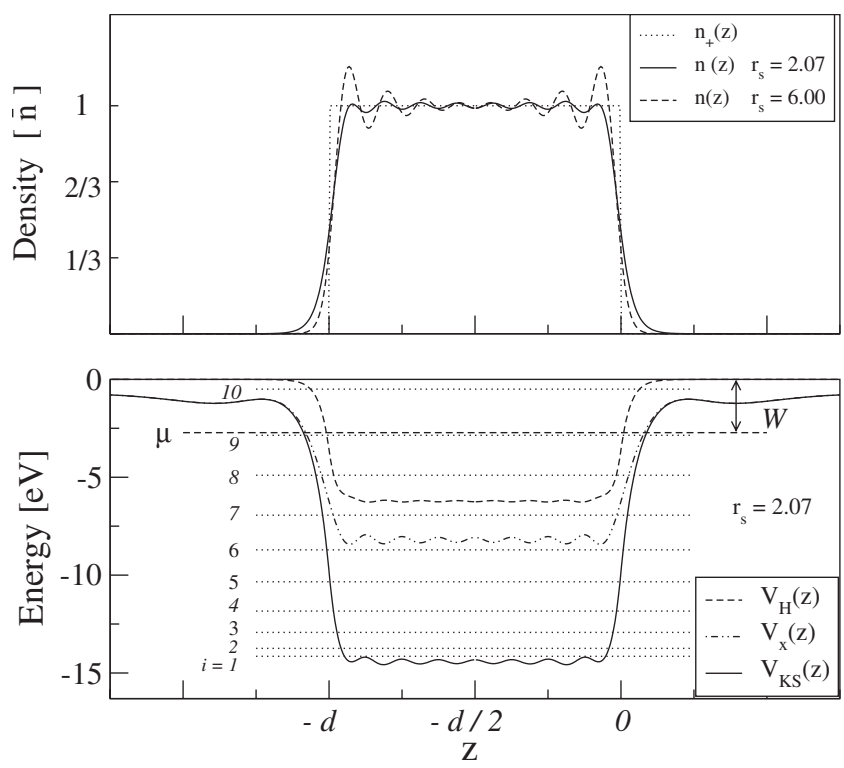

FIG. 1. Main features of the jellium-slab model of metal surfaces. Top panel: normalized jellium density $\left[n_{+}(z)\right]$ and the selfconsistent OEP electron density $n(z)$ for two different values of the electron-density parameter $r_{s}$. Lower panel: OEP Hartree, exchange, and Kohn-Sham potentials for $r_{s}=2.07$. Dotted lines denote KS eigenvalues, $\varepsilon_{F}$ is the Fermi energy, and $W$ is the work function. $d=4 \lambda_{F}$.

ions inside the metal is replaced by a uniform distribution of positive charge (the jellium). The positive jellium density is defined as

$$
n_{+}(z)=\bar{n} \theta\left(\frac{d}{2}-\left|z+\frac{d}{2}\right|\right),
$$

which describes a slab of width $d$, number density $\bar{n},{ }^{30}$ and jellium edges at $z=-d$ and $z=0 . \theta(x)$ represents the Heaviside step function: $\theta(x)=1$ if $x>0$ and $\theta(x)=0$ if $x<0$. A schematic view of our jellium slab is given in Fig. 1. Besides, and for convenience for the numerical calculations, infinite barriers are located far from the jellium edges, well inside the left and right evanescent vacuum regions. We have checked that these infinite barriers are located far enough for all the numerical calculations presented here to be independent of their precise location. ${ }^{31}$ The jellium-slab model is invariant under translations in the $x-y$ plane, so the KS eigenfunctions can be factorized as follows:

$$
\varphi_{i, \mathbf{k}}(\mathbf{r})=\frac{e^{i \mathbf{k} \cdot \rho}}{\sqrt{A}} \xi_{i}(z),
$$

where $\rho$ and $\mathbf{k}$ are the in-plane coordinate and wave vector, respectively, and $A$ represents a normalization area. $\xi_{i}(z)$ are the normalized spin-degenerate eigenfunctions for electrons in slab discrete levels (SDLs) $i(i=1,2, \ldots)$ with energy $\varepsilon_{i}$. They are the solutions of the effective one-dimensional KS equation,

$$
\hat{h}_{\mathrm{KS}}^{i}(z) \xi_{i}(z)=\left[-\frac{\hbar^{2}}{2 m_{e}} \frac{\partial^{2}}{\partial z^{2}}+V_{\mathrm{KS}}(z)-\varepsilon_{i}\right] \xi_{i}(z)=0,
$$

with $m_{e}$ as the bare electron mass.

The KS potential $V_{\mathrm{KS}}$ entering Eq. (3) is the sum of two distinct contributions,

$$
V_{\mathrm{KS}}(z)=V_{H}(z)+V_{\mathrm{xc}}(z),
$$

where $V_{H}(z)$ is the classical (electrostatic) Hartree potential, given by ${ }^{32}$

$$
V_{H}(z)=-2 \pi e^{2} \int_{-\infty}^{\infty} d z^{\prime}\left|z-z^{\prime}\right|\left[n\left(z^{\prime}\right)-n_{+}\left(z^{\prime}\right)\right] .
$$

Here, $n(z)$ is the electron number density, ${ }^{33}$

$$
n(z)=\frac{1}{2 \pi} \sum_{i}^{\text {occ. }}\left(k_{F}^{i}\right)^{2}\left|\xi_{i}(z)\right|^{2},
$$

where $k_{F}^{i}=\sqrt{2 m_{e}\left(\mu-\varepsilon_{i}\right)} / \hbar$, and $\mu=\mu(\bar{n}, d)$ is the chemical potential, which in turn is determined from the neutrality condition for the whole system by the condition $\sum_{i}^{\text {occ. }}\left(k_{F}^{i}\right)^{2}$ $=2 \pi d \bar{n} . V_{\mathrm{xc}}(z)$ is the nonclassical xc potential, which is obtained as the functional derivative of the so-called xc energy functional $E_{\mathrm{xc}}[n(z)],{ }^{34}$

$$
V_{\mathrm{xc}}(z) \equiv \frac{1}{A} \frac{\delta E_{\mathrm{xc}}[n(z)]}{\delta n(z)} .
$$

Applications of DFT typically proceed from explicit density-dependent forms of $E_{\mathrm{xc}}$, as obtained using a variety of local or semilocal approximations. However, in the last few years increasing attention has been devoted to orbitaldependent forms of $E_{\mathrm{xc}}: E_{\mathrm{xc}}=E_{\mathrm{xc}}\left[\left\{\xi_{i}\right\},\left\{\varepsilon_{i}\right\}\right]$, which are only implicit functionals of the electron density $n(z)$. In this case, one resorts to the OEP method ${ }^{16}$ or, equivalently, uses repeatedly the chain rule for functional derivatives to obtain the following expression for the xc potential of Eq. (7) (Ref. 35):

$$
\begin{aligned}
V_{\mathrm{xc}}(z)= & \frac{1}{A} \sum_{i}^{\text {occ. }} \int_{-\infty}^{\infty} d z^{\prime} \int_{-\infty}^{\infty} d z^{\prime \prime} \\
& \times\left[\frac{\delta E_{\mathrm{xc}}}{\delta \xi_{i}\left(z^{\prime \prime}\right)} \frac{\delta \xi_{i}\left(z^{\prime \prime}\right)}{\delta V_{\mathrm{KS}}\left(z^{\prime}\right)}+\text { c.c. }\right] \frac{\delta V_{\mathrm{KS}}\left(z^{\prime}\right)}{\delta n(z)} .
\end{aligned}
$$

Multiplying Eq. (8) by the $\mathrm{KS}$ density-response function $\chi_{\mathrm{KS}}\left(z, z^{\prime}\right) \equiv \delta n(z) / \delta V_{\mathrm{KS}}\left(z^{\prime}\right)$, using the identity

$$
\int_{-\infty}^{\infty} \chi_{\mathrm{KS}}\left(z, z^{\prime}\right) \chi_{\mathrm{KS}}^{-1}\left(z^{\prime}, z^{\prime \prime}\right) d z=\delta\left(z-z^{\prime \prime}\right),
$$

comparing Eqs. (7) and (8), and integrating over the coordinate $z$, one finds

$$
\begin{aligned}
\int_{-\infty}^{\infty} & \frac{\delta E_{\mathrm{xc}}}{\delta n\left(z^{\prime}\right)} \chi_{\mathrm{KS}}\left(z, z^{\prime}\right) d z^{\prime} \\
= & \sum_{i}^{\text {occ. }} \int_{-\infty}^{\infty}\left[\frac{\delta E_{\mathrm{xc}}}{\delta \xi_{i}\left(z^{\prime}\right)} \frac{\delta \xi_{i}\left(z^{\prime}\right)}{\delta V_{\mathrm{KS}}(z)}+\text { c.c. }\right] d z^{\prime} .
\end{aligned}
$$


The nice feature of Eq. (10) is that $\delta \xi_{i}\left(z^{\prime}\right) / \delta V_{\mathrm{KS}}(z)$ and $\chi_{\mathrm{KS}}\left(z, z^{\prime}\right)$ are simply obtained from the solutions of Eq. (3) as follows:

$$
\frac{\delta \xi_{i}\left(z^{\prime}\right)}{\delta V_{\mathrm{KS}}(z)}=\xi_{i}(z) \sum_{j(\neq i)} \frac{\xi_{j}\left(z^{\prime}\right)^{*} \xi_{j}(z)}{\left(\varepsilon_{i}-\varepsilon_{j}\right)} \equiv \xi_{i}(z) G_{i}^{\mathrm{KS}}\left(z^{\prime}, z\right),
$$

and

$$
\begin{gathered}
\chi_{\mathrm{KS}}\left(z, z^{\prime}\right)=\sum_{i}^{\text {occ. }} \int_{-\infty}^{\infty} d z^{\prime \prime}\left[\frac{\delta n(z)}{\delta \xi_{i}\left(z^{\prime \prime}\right)} \frac{\delta \xi_{i}\left(z^{\prime \prime}\right)}{\delta V_{\mathrm{KS}}\left(z^{\prime}\right)}+\text { c.c. }\right], \\
=\frac{1}{4 \pi} \sum_{i}^{\text {occ. }}\left[\left(k_{F}^{i}\right)^{2} \xi_{i}(z)^{*} \xi_{i}\left(z^{\prime}\right) G_{i}^{\mathrm{KS}}\left(z^{\prime}, z\right)+\text { c.c. }\right],
\end{gathered}
$$

where $G_{i}^{\mathrm{KS}}\left(z^{\prime}, z\right)$ is Green's function of noninteracting $\mathrm{KS}$ electrons. In the calculation of $\chi_{\mathrm{KS}}\left(z, z^{\prime}\right)$, the chain rule for functional derivatives has been used; now we are considering the density itself as a functional of the occupied SDL. In obtaining Eq. (13) from Eq. (12), we have used Eq. (11) and also that

$$
\frac{\delta n(z)}{\delta \xi_{i}\left(z^{\prime}\right)}=\delta\left(z-z^{\prime}\right) \frac{\left(k_{F}^{i}\right)^{2}}{2 \pi} \xi_{i}(z)^{*}
$$

which follows from Eq. (6).

Introducing Eqs. (11) and (13) into the central Eq. (10), we obtain the final and compact version of the OEP integral equation for $V_{\mathrm{xc}}(z)$,

$$
\sum_{i}^{\text {occ. }} S_{i}(z)=0
$$

where

$$
S_{i}(z)=\left(k_{F}^{i}\right)^{2} \Psi_{i}(z)^{*} \xi_{i}(z)+\text { c.c. }
$$

and

$$
\Psi_{i}(z)=\sum_{j(\neq i)} \frac{\xi_{j}(z)}{\left(\varepsilon_{i}-\varepsilon_{j}\right)} \int_{-\infty}^{\infty} \xi_{j}\left(z^{\prime}\right)^{*} \Delta V_{\mathrm{xc}}^{i}\left(z^{\prime}\right) \xi_{i}\left(z^{\prime}\right) d z^{\prime} .
$$

Here, $\quad \Delta V_{\mathrm{xc}}^{i}(z)=V_{\mathrm{xc}}(z)-u_{\mathrm{xc}}^{i}(z)$, where $u_{\mathrm{xc}}^{i}(z)$ are SDLdependent xc potentials of the form ${ }^{36}$

$$
u_{\mathrm{xc}}^{i}(z) \equiv\left[4 \pi / A\left(k_{F}^{i}\right)^{2} \xi_{i}(z)^{*}\right] \delta E_{\mathrm{xc}} \delta \xi_{i}(z) .
$$

The magnitudes $\Psi_{i}(z)$ are called the "shifts," as they can be physically interpreted as the first-order corrections of the KS eigenfunctions $\xi_{i}(z)$ under the perturbation $\Delta V_{\mathrm{xc}}^{i}(z)$. These shifts also provide a useful and practical tool for the numerical solution of the OEP equation. ${ }^{37,38}$ From Eq. (15), we find the orthogonality constraint between the KS eigenfunctions and the shifts: $\int \xi_{i}(z)^{*} \Psi_{i}(z) d z=0$. It is also immediate that the shifts are invariant under the replacement $V_{\mathrm{xc}}(z) \rightarrow V_{\mathrm{xc}}(z)+\alpha$, with $\alpha$ being an arbitrary constant. This means that the above set of equations determines $V_{\text {xc }}(z)$ up to an additive constant, which should be fixed by imposing a suitable boundary condition. Moreover, the shifts $\Psi_{i}(z)$ are easily found to satisfy the following inhomogeneous differential equation: ${ }^{20}$

$$
\hat{h}_{\mathrm{KS}}^{i}(z) \Psi_{i}(z)=-\left[\Delta V_{\mathrm{xc}}^{i}(z)-\Delta \bar{V}_{\mathrm{xc}}^{i}\right] \xi_{i}(z) .
$$

Here, mean values are defined as $\bar{O}^{i}=\int \xi_{i}(z)^{*} O^{i}(z) \xi_{i}(z) d z$.

Equations (3)-(5) and (14), which determine the local $V_{\mathrm{xc}}(z)$ corresponding to a given SDL-dependent $E_{\mathrm{xc}}$, form a closed system of equations (the OEP equations), which should be solved in a self-consistent way. In order to accomplish some contact with other useful versions of the OEP equations for the present problem, a few additional steps are required. First of all, we write

$$
\xi_{i}(z)^{*} \hat{h}_{\mathrm{KS}}^{i}(z) \Psi_{i}(z)=-\frac{\hbar^{2}}{2 m_{e}}\left[\xi_{i}(z)^{*} \frac{\partial^{2} \Psi_{i}(z)}{\partial z^{2}}-\Psi_{i}(z) \frac{\partial^{2} \xi_{i}(z)^{*}}{\partial z^{2}}\right],
$$

which is easily obtained from Eq. (3). Second, we multiply the left-hand side of Eq. (16) by $\xi_{i}(z)^{*}$ to obtain

$$
\begin{gathered}
\frac{\hbar^{2}}{2 m_{e}}\left[\xi_{i}(z)^{*} \frac{\partial^{2} \Psi_{i}(z)}{\partial z^{2}}-\Psi_{i}(z) \frac{\partial^{2} \xi_{i}(z)^{*}}{\partial z^{2}}\right] \\
=\left[\Delta V_{\mathrm{xc}}^{i}(z)-\Delta \bar{V}_{\mathrm{xc}}^{i}\right]\left|\xi_{i}(z)\right|^{2} .
\end{gathered}
$$

Then, we start from the self-evident identity,

$$
\begin{aligned}
V_{\mathrm{xc}}(z)= & \sum_{i}^{\text {occ. }} \frac{\left(k_{F}^{i}\right)^{2}\left|\xi_{i}(z)\right|^{2}}{4 \pi n(z)} \\
& \times\left[u_{\mathrm{xc}}^{i}(z)+\Delta \bar{V}_{\mathrm{xc}}^{i}+\Delta V_{\mathrm{xc}}^{i}(z)-\Delta \bar{V}_{\mathrm{xc}}^{i}+\text { c.c. }\right],
\end{aligned}
$$

we eliminate the factor $\left[\Delta V_{\mathrm{xc}}^{i}(z)-\Delta \bar{V}_{\mathrm{xc}}^{i}\right]\left|\xi_{i}(z)\right|^{2}$ by using Eq. (17), and we obtain

$$
\begin{aligned}
V_{\mathrm{xc}}(z)= & \sum_{i} \frac{\left(k_{F}^{i}\right)^{2}}{4 \pi n(z)}\left\{\left|\xi_{i}(z)\right|^{2}\left[u_{\mathrm{xc}}^{i}(z)+\Delta \bar{V}_{\mathrm{xc}}^{i}\right]\right. \\
& \left.+\frac{\hbar^{2}}{2 m_{e}}\left[\xi_{i}(z)^{*} \frac{\partial^{2} \Psi_{i}(z)}{\partial z^{2}}-\Psi_{i}(z) \frac{\partial^{2} \xi_{i}(z)^{*}}{\partial z^{2}}\right]+\text { c.c. }\right\} .
\end{aligned}
$$

Finally, we proceed with the elimination from Eq. (19) of the term proportional to $\partial^{2} \Psi_{i}(z) / \partial z^{2}$, the subsequent elimination of $\partial^{2} \xi_{i}(z)^{*} / \partial z^{2}$ proceeds via the KS equations, and as a result of all these manipulations we obtain the following expression for the DFT xc potential: ${ }^{39}$

$$
V_{\mathrm{xc}}(z)=V_{\mathrm{xc}, 1}(z)+V_{\mathrm{xc}, 2}(z),
$$

where

$$
V_{\mathrm{xc}, 1}(z)=\sum_{i}^{\mathrm{occ} .} \frac{\left(k_{F}^{i}\right)^{2}\left|\xi_{i}(z)\right|^{2}}{4 \pi n(z)}\left\{u_{\mathrm{xc}}^{i}(z)+\Delta \bar{V}_{\mathrm{xc}}^{i}+\text { c.c. }\right\}
$$

and

$$
\begin{aligned}
V_{\mathrm{xc}, 2}(z)= & -\frac{1}{2 \pi n(z)} \sum_{i}^{\text {occ. }}\left(\mu-\varepsilon_{i}\right)\left[\left(k_{F}^{i}\right)^{2} \Psi_{i}(z) \xi_{i}(z)^{*}\right. \\
& \left.+\Psi_{i}^{\prime}(z) \xi_{i}^{\prime}(z)^{*}+\text { c.c. }\right],
\end{aligned}
$$

with primes denoting derivatives with respect to the $z$ coordinate. It is important to note that Eqs. (14) and (20) are just two different, but fully equivalent, ways to obtain the OEP 
xc potential for the present problem. If the shifts $\Psi_{i}(z)$ are (arbitrarily) forced to be identically equal to zero, the only term that survives is $V_{\mathrm{xc}, 1}(z)$. This is exactly the Krieger-Li-Iafrate $^{24}$ (KLI) approximation, which brings the identification $V_{\mathrm{xc}, 1}(z) \equiv V_{\mathrm{xc}}^{\mathrm{KLI}}(z) .{ }^{40}$ As before, Eqs. (3)-(5) and (20) form a closed set of equations, which should be solved self-consistently.

Both exchange and correlation have been included so far. Unless stated otherwise, we will now focus on the $x$-only case, where $E_{\mathrm{xc}}, V_{\mathrm{xc}}(z)$, and $u_{\mathrm{xc}}(z)$ are replaced by $E_{x}, V_{x}(z)$, and $u_{x}(z)$, respectively. We have achieved the self-consistent numerical solution of the $x$-only version of the OEP equations by two different methods: (i) direct calculation of the shifts of Eq. (15), by solving Eq. (16), ${ }^{37}$ and (ii) direct solution of the OEP integral equation for $V_{x}(z)$, as given by the $x$-only version of Eq. (14). ${ }^{38}$ Both methods yield results that agree within numerical accuracy, although the first approach is found to be computationally more efficient than the second. Both methods face numerical instabilities beyond a critical coordinate $z$ in the vacuum region.

Finally, we note that the exact-exchange energy of a jellium slab is given by the following expression:

$$
E_{x}(d)=\frac{A}{4 \pi} \sum_{i}^{\text {occ. }}\left(k_{F}^{i}\right)^{2} \int_{-\infty}^{\infty} d z\left|\xi_{i}(z)\right|^{2} u_{x}^{i}(z)
$$

where $u_{x}^{i}(z)$ represent the SDL-dependent exchange potentials,

$$
u_{x}^{i}(z)=-\frac{2 e^{2}}{\left(k_{F}^{i}\right)^{2}} \sum_{j}^{\text {occ. }} \frac{\xi_{j}(z)^{*}}{\xi_{i}(z)^{*}} \int_{-\infty}^{\infty} d z^{\prime} \frac{\xi_{i}\left(z^{\prime}\right)^{*} g\left(\Delta z k_{F}^{i}, \Delta z k_{F}^{j}\right) \xi_{j}\left(z^{\prime}\right)}{(\Delta z)^{3}}
$$

with $\Delta z=\left|z-z^{\prime}\right|$,

$$
g\left(s, s^{\prime}\right)=s s^{\prime} \int_{0}^{\infty} \frac{J_{1}(s t) J_{1}\left(s^{\prime} t\right)}{\sqrt{1+t^{2}}} \frac{d t}{t},
$$

being the "universal" (that is, independent of $V_{\mathrm{KS}}$ ) function introduced by Kohn and Mattsson ${ }^{41}$ and $J_{1}(x)$ being the firstorder cylindrical Bessel function. ${ }^{42}$

\section{ASYMPTOTICS OF THE EXACT-EXCHANGE KS POTENTIAL}

The long-range behavior of $V_{\mathrm{xc}}(z)$ in the vacuum region is an important and open issue in DFT studies of metal surfaces. ${ }^{43}$ The aim of this section is to present a detailed derivation of the analytical asymptotic limit of $V_{x}(z)$ reported in Ref. 39 for a slab geometry. First of all, we note that by making the choice that $V_{\mathrm{KS}}(z \rightarrow \infty) \rightarrow 0$, Eq. (3) leads us to the conclusion that $\xi_{i}(z \rightarrow \infty) \rightarrow e^{-z \sqrt{-2 m_{e} \varepsilon_{i}} / \hbar}$ for all occupied $i$ (disregarding a factor involving powers of $z$ ). We also remark the following points: (i) due to the exponential decay of $V_{H}(z \rightarrow \infty)$, the assumption $V_{\mathrm{KS}}(z \rightarrow \infty) \rightarrow 0$ implies that $V_{x}(z \rightarrow \infty) \rightarrow 0$; (ii) for this choice of the zero of energy, one finds $\varepsilon_{i}<0$ for all occupied states; (iii) the slowest decaying of all the occupied SDL corresponds to $i=m$, where $m$ is the highest occupied SDL.
Now we look at the asymptotic behavior of the shifts $\Psi_{i}(z)$. Turning to the $x$-only version of Eq. (16),

$$
\begin{aligned}
& {\left[-\frac{\hbar^{2}}{2 m_{e}} \frac{\partial^{2}}{\partial z^{2}}+V_{H}(z)+V_{x}(z)-\varepsilon_{i}\right] \Psi_{i}(z)} \\
& =-V_{x}(z) \xi_{i}(z)+u_{x}^{i}(z) \xi_{i}(z)+\Delta \bar{V}_{x}^{i} \xi_{i}(z),
\end{aligned}
$$

we focus on the asymptotic behavior of the three terms on the right-hand side (rhs) of this equation, ${ }^{20}$

$$
\begin{gathered}
V_{x}(z \rightarrow \infty) \xi_{i}(z \rightarrow \infty) \rightarrow V_{x}(z \rightarrow \infty) e^{-z \beta_{i}}, \\
u_{x}^{i}(z \rightarrow \infty) \xi_{i}(z \rightarrow \infty) \rightarrow e^{-z \beta_{m}}, \\
\Delta \bar{V}_{x}^{i} \xi_{i}(z \rightarrow \infty) \rightarrow e^{-z \beta_{i}},
\end{gathered}
$$

with $\beta_{i}=\sqrt{-2 m_{e} \varepsilon_{i}} / \hbar$. Equation (26) follows from an inspection of Eq. (22) in the limit $z \rightarrow \infty$; in this limit, the sum over $j$ is exponentially dominated by the term $j=m$, and the result of Eq. (26) follows at once. Hence, for $i \neq m$ Eq. (24) yields

$$
\left[-\frac{\hbar^{2}}{2 m_{e}} \frac{\partial^{2}}{\partial z^{2}}-\varepsilon_{i}\right] \Psi_{i}(z \rightarrow \infty) \rightarrow e^{-z \beta_{m}},
$$

i.e., $\Psi_{i}(z \rightarrow \infty) \rightarrow e^{-z \beta_{m}}$. For $i=m$, all three terms in the rhs of Eq. (24) decay equally (to exponential accuracy), and further analysis is necessary. Equation (14) can be rewritten as follows,

$$
\left(k_{F}^{m}\right)^{2} \Psi_{m}(z)^{*} \xi_{m}(z)+\text { c.c. }=-\sum_{i=1}^{m-1}\left(k_{F}^{i}\right)^{2} \Psi_{i}(z)^{*} \xi_{i}(z)-\text { c.c. },
$$

and by studying its asymptotic limit, it is clear that its rhs can be approximated by the term $i=m-1$ (with exponential accuracy). Given that both $\xi_{m}(z \rightarrow \infty)$ and $\Psi_{m-1}(z \rightarrow \infty)$ decay as $e^{-z \beta_{m-1}}$, it follows that $\Psi_{m}(z \rightarrow \infty)$ decays as $\xi_{m-1}(z \rightarrow \infty)$, that is, $\Psi_{m}(z \rightarrow \infty) \rightarrow e^{-z \beta_{m-1}}$. Armed with these results, the asymptotic limit of $V_{x}(z)$ is immediate from Eq. (20): $V_{x, 2}(z \rightarrow \infty)$ tends exponentially to zero, while

$$
V_{x, 1}(z \rightarrow \infty) \rightarrow u_{x}^{m}(z \rightarrow \infty)+\Delta \bar{V}_{x}^{m} .
$$

The leading contribution to $u_{x}^{m}(z \rightarrow \infty)$ is easily obtained from Eq. (22) by considering once again that in this regime the sum over $j$ is exponentially dominated by the term $j=m$. For this case, the integral over the coordinate $t$ can be evaluated analytically, yielding

$$
\begin{aligned}
u_{x}^{m}(z \rightarrow \infty) \rightarrow & -e^{2} \int_{-\infty}^{\infty} \frac{\left|\xi_{m}\left(z^{\prime}\right)\right|^{2}}{\left|z-z^{\prime}\right|} d z^{\prime} \\
& \times\left[1-\frac{I_{1}\left(2 k_{F}^{m}\left|z-z^{\prime}\right|\right)}{k_{F}^{m}\left|z-z^{\prime}\right|}+\frac{L_{1}\left(2 k_{F}^{m}\left|z-z^{\prime}\right|\right)}{k_{F}^{m}\left|z-z^{\prime}\right|}\right],
\end{aligned}
$$

where $I_{1}$ and $L_{1}$ are the modified Bessel and Struve functions, respectively. ${ }^{42}$ Noting now that in this regime $k_{F}^{m} \mid z$ $-z^{\prime} \mid \simeq k_{F}^{m} z \gg 1,{ }^{44}$ it is permissible to expand the integrand of Eq. (31) as follows: 


$$
\begin{aligned}
u_{x}^{m}(z \rightarrow \infty) \rightarrow & -\frac{e^{2}}{z} \int_{-\infty}^{\infty}\left|\xi_{m}\left(z^{\prime}\right)\right|^{2} d z^{\prime} \\
& \times\left[1+\frac{z^{\prime}}{z}-\frac{2}{\pi k_{F}^{m} z}+O\left(\frac{1}{z^{2}}\right)\right] .
\end{aligned}
$$

Using the normalization of the orbitals $\xi_{m}(z)$, we obtain

$$
u_{x}^{m}(z \rightarrow \infty) \rightarrow-\frac{e^{2}}{z}\left(1+\frac{\beta}{z}+\cdots\right),
$$

with $\beta=\bar{z}^{m}-2 /\left(\pi k_{F}^{m}\right) .{ }^{45}$ Since the exchange potential $V_{x}(z)$ has been chosen to vanish at large distances from the surface into the vacuum $\left[V_{x}(\infty)=0\right]$, Eq. (30) leads us to the important constraint,

$$
\Delta \bar{V}_{x}^{m}=\bar{V}_{x}^{m}-\bar{u}_{x}^{m}=0
$$

which fixes the undetermined constant in $V_{x}(z)$ discussed above. All numerical results presented here have been obtained by using this constraint. From Eqs. (30), (33), and (34), we conclude that

$$
\begin{aligned}
V_{x}(z \rightarrow \infty) & \rightarrow V_{x, 1}(z \rightarrow \infty) \rightarrow u_{x}^{m}(z \rightarrow \infty) \\
& \rightarrow-\frac{e^{2}}{z}\left(1+\frac{\beta}{z}+\cdots\right),
\end{aligned}
$$

which is the main result of this section.

At this point, we emphasize that the asymptotics dictated by Eq. (35) hold only at $z$ coordinates that are larger than $1 / k_{F}^{m}$. As $k_{F}^{m}$ is of the order of $1 / d$ (or smaller, depending on the actual value of $d$ ), Eq. (35) shows that the $x$-only KS potential happens to be four times larger than the classical image potential $\left[V_{\mathrm{im}}(z)=-e^{2} / 4 z\right]$ only at a distance $z$ that is considerably larger than the slab thickness. Furthermore, the arguments leading to Eqs. (26) and (31) are only valid for a discrete slab spectrum, such that there is a finite-energy-gap between $\varepsilon_{m}$ and the remaining occupied energy levels $\varepsilon_{i}(i$ $<m$ ). An extension of the present OEP framework to treat the case of a semi-infinite jellium surface ${ }^{46}$ is now in progress. $^{47}$

Finally, we note that under the condition $V_{\mathrm{KS}}(\infty)=0 \mathrm{Eq}$. (35) for the asymptotics of $V_{x}(z)$ remains valid when correlation is included in the evaluation of the shifts $\Psi_{i}(z)$. The point here is that the shifts are separable in their exchange and correlation components, and they also satisfy separated differential equations [like Eq. (24) for exchange]. Once exchange and correlation contributions are split, the analysis of the asymptotic behavior of $V_{x}(z)$ follows the same lines as above, and the asymptotic limit of Eq. (35) remains the same.

\section{SURFACE ENERGY}

In this section, surface-energy calculations are presented, as obtained at the $x$-only level. The surface energy $\sigma$ is the work required, per unit area of the new surface formed, to split the crystal in two along a plane. ${ }^{1}$ For our slab geometry,

$$
\sigma(d)=\frac{2 E(d)-E(2 d)}{2 A}
$$

where $E(d)$ is the total ground-state energy for each half of the slab after it is split (width $d$ ), and $E(2 d)$ is the total ground-state energy of the unsplit slab (width $2 d$ ), both the split and unsplit systems with the same jellium density.

Following the standard DFT energy-functional partitioning, the surface energy (without correlation contribution) can be written as the sum of three terms, ${ }^{6}$

$$
\sigma(d)=\sigma_{K}(d)+\sigma_{\mathrm{el}}(d)+\sigma_{x}(d),
$$

where $\sigma_{K}(d)$ is the noninteracting kinetic contribution to the surface energy, $\sigma_{\mathrm{el}}(d)$ is the electrostatic surface energy due to all noncompensated positive and negative charge distributions in the slab, and $\sigma_{x}(d)$ is the exchange contribution to the surface energy. From elementary physical arguments, it follows that $\sigma_{K}(d)<0$, while $\sigma_{\text {el }}(d)$ and $\sigma_{x}(d)$ are both positive. $^{2}$ Also, the stability of the slab against spontaneous fragmentation is accomplished if $\sigma(d)>0$. From Eqs. (36) and (37), one writes

$$
\sigma_{l}(d)=\left[2 E_{l}(d)-E_{l}(2 d)\right] /(2 A),
$$

with $l=K, \mathrm{el}, x$, and

$$
E_{K}(d)=\frac{A \hbar^{2}}{4 \pi m_{e}} \sum_{i}^{\text {occ. }}\left(k_{F}^{i}\right)^{2}\left[\frac{\left(k_{F}^{i}\right)^{2}}{2}-\int_{-\infty}^{\infty} \xi_{i}(z) \frac{\partial^{2} \xi_{i}(z)}{\partial z^{2}} d z\right],
$$

$$
E_{\mathrm{el}}(d)=\frac{A}{2} \int_{-\infty}^{\infty} V_{H}(z)\left[n(z)-n_{+}(z)\right] d z,
$$

and [see Eqs. (21) and (22)]

$$
\begin{aligned}
E_{x}(d)= & -\frac{e^{2} A}{2 \pi} \sum_{i, j}^{\text {occ. }} \int_{-\infty}^{\infty} d z \int_{-\infty}^{\infty} d z^{\prime} \\
& \times \frac{\xi_{i}(z)^{*} \xi_{j}\left(z^{\prime}\right)^{*} g\left(k_{F}^{i} \Delta z, k_{F}^{j} \Delta z\right) \xi_{j}(z) \xi_{i}\left(z^{\prime}\right)}{(\Delta z)^{3}} .
\end{aligned}
$$

The dependence on the slab width $d$ in Eqs. (39)-(41) enters through the self-consistent KS eigenvalues $\left(\varepsilon_{i}\right)$ and eigenfunctions $\left[\xi_{i}(z)\right]$.

Alternatively, one can define the effective single-slab surface energies, ${ }^{48}$

$$
\sigma(d)=\frac{E(d)-E^{\mathrm{unif}}(d)}{2 A}
$$

and

$$
\sigma_{l}(d)=\left[E_{l}(d)-E_{l}^{\mathrm{unif}}(d)\right] /(2 A),
$$

where $E_{l}^{\text {unif }}(d)$ is the ground-state energy of a uniform slab of electron gas of size $d$ and $l=K$, el, $x .^{49}$ Note that Eq. (42) only reproduces the surface-energy definition of Eq. (36) as $d \rightarrow \infty$. However, for a correct extrapolation of finite-slab calculations to the infinite-width limit, ${ }^{48}$ here we calculate numerically the three components of the surface energy from the single-slab [Eq. (43)]. We have checked that the differ- 


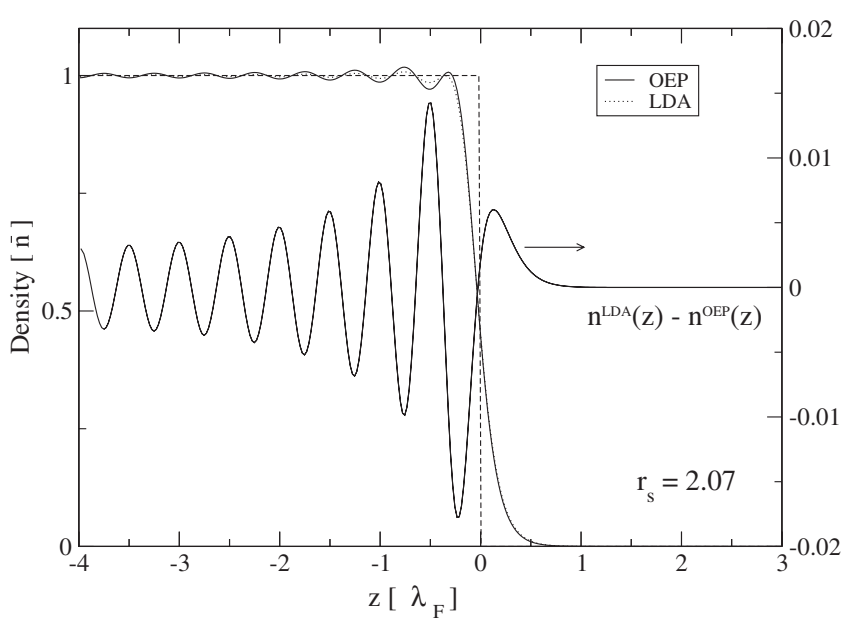

FIG. 2. LDA and OEP self-consistent electron densities and its difference for $d=8 \lambda_{F}$ and $r_{s}=2.07$. Note that $n^{\mathrm{LDA}}(z)$ is slightly more diffuse than $n^{\mathrm{OEP}}(z)$, as $n^{\mathrm{LDA}}(z)-n^{\mathrm{OEP}}(z)>0$ for $z$ outside the jellium edge (in the vacuum).

ences between surface energies obtained from Eqs. (36) and (42) are quite small even for the narrowest slabs studied and that both agree in the extrapolation toward the semi-infinite limit.

Being the ground-state density the basic ingredient of DFT, we found interesting to compare the differences between the different density profiles that we have obtained. We exhibit in Fig. 2 the self-consistent electron-density profiles that we have obtained within the $x$-only LDA and OEP schemes for $r_{s}=2.07$ and $d=8 \lambda_{F}{ }^{50}$ It is expected that the amplitude of the difference between both densities diminishes as $z$ approaches the slab center, where both $n^{\mathrm{LDA}}(z \rightarrow-d / 2)$ and $n^{\mathrm{OEP}}(z \rightarrow-d / 2)$ should approach $\bar{n}$ as $d \rightarrow \infty$. Figure 2 shows that there are noticeable differences between both densities: $n^{\mathrm{LDA}}(z)$ extends further into the vacuum region than $n^{\mathrm{OEP}}(z)$, which is a result of the LDA orbitals being more extended or "diffuse" than their OEP counterparts, and the amplitude of the Friedel oscillations near the surface is larger for $n^{\mathrm{OEP}}(z)$ than for $n^{\mathrm{LDA}}(z)$. We have found the same behavior for other values of $r_{s}$.

Figure 3 shows the results that we have obtained for the slab kinetic surface energy, as a function of the slab width $d$, for $r_{s}=2.07$. As in the case of the electron density, we have performed these calculations within the $x$-only LDA and OEP schemes. In the LDA, the kinetic surface energy $\sigma_{K}(d)$ (LDA) is obtained by introducing the $x$-only self-consistent LDA eigenfunctions $\xi_{i}^{\mathrm{LDA}}(z)$ and eigenvalues $\varepsilon_{i}^{\mathrm{LDA}}$ into the formally exact Eq. (39). In the OEP, the kinetic surface energy $\sigma_{K}(d)(\mathrm{OEP})$ is obtained by using the same equation [Eq. (39)] but with the LDA eigenfunctions and eigenvalues replaced by their $x$-only OEP counterparts $\xi_{i}^{\mathrm{OEP}}(z)$ and $\varepsilon_{i}^{\mathrm{OEP}}$. The strong oscillations in both $\sigma_{K}(d)$ (LDA) and $\sigma_{K}(d)$ (OEP) are the result of the sequential filling of empty slab discrete levels as $d$ increases. Maxima in $\sigma_{K}(d)$ correspond to the onset for the filling of a new slab discrete level. For this particular case, and following the extrapolation procedure of Ref. 48, we have obtained the infinite-width extrapolated surface energies $\sigma_{K}(\mathrm{LDA})=-4832 \mathrm{erg} / \mathrm{cm}^{2}$ (as reported in Ref. 48) and $\sigma_{K}(\mathrm{OEP})=-4720 \mathrm{erg} / \mathrm{cm}^{2}$.

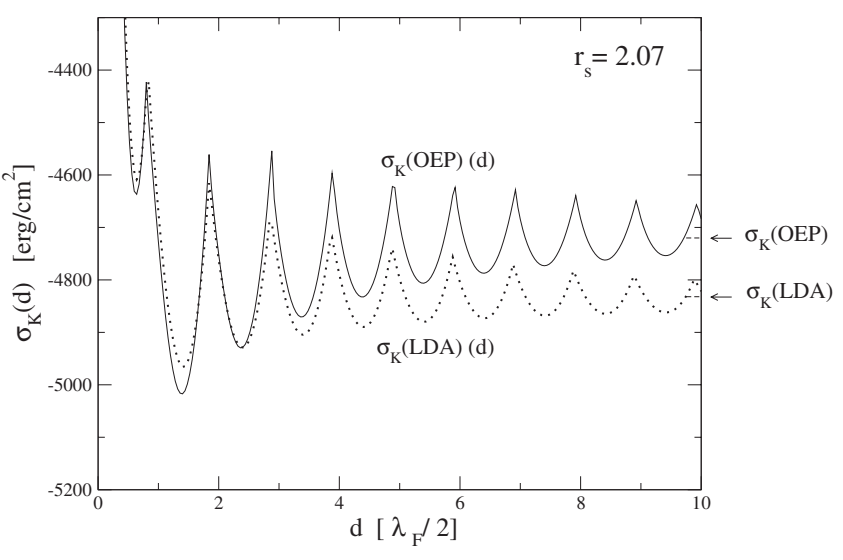

FIG. 3. Kinetic surface energy, as a function of slab width $d$, for $r_{s}=2.07$, from Eq. (43), with $l=K$. Full line, OEP results; dotted line, LDA results. The two arrows on the right denote the extrapolated asymptotic values $\sigma_{K}(\mathrm{OEP}) \rightarrow-4720 \mathrm{erg} / \mathrm{cm}^{2}, \sigma_{K}(\mathrm{LDA}) \rightarrow$ $-4832 \mathrm{erg} / \mathrm{cm}^{2}$.

Figure 4 displays the results that we have obtained for the electrostatic contribution to the surface energy, as a function of the slab width $d$ and for $r_{s}=2.07$, again within the $x$-only LDA and OEP schemes. The electrostatic surface energies $\sigma_{\mathrm{el}}(d)(\mathrm{LDA})$ and $\sigma_{\mathrm{el}}(d)(\mathrm{OEP})$ are obtained from Eq. (40) by using either the $x$-only LDA electron density $n^{\mathrm{LDA}}(z)$ or the $x$-only OEP electron density $n^{\mathrm{OEP}}(z)$, respectively. In this case, the onset for the filling of a new slab discrete level is always associated with a minimum. Following the extrapolation procedure of Ref. 48, we have obtained the infinitewidth surface energies indicated by arrows in Fig. 4: $\sigma_{\mathrm{el}}(\mathrm{LDA})=1172 \mathrm{erg} / \mathrm{cm}^{2}$ (as reported in Ref. 48) and $\sigma_{\text {el }}(\mathrm{OEP})=1103 \mathrm{erg} / \mathrm{cm}^{2}$.

In Fig. 5, we show the results that we have obtained for the exact-exchange contribution to the slab surface energy, as a function of the slab width $d$ and for $r_{s}=2.07$, again within the $x$-only LDA and OEP schemes. As in the case of the kinetic and electrostatic surface energies, exact-exchange surface energies $\sigma_{x}(d)(\mathrm{LDA})$ and $\sigma_{x}(d)(\mathrm{OEP})$ [both derived

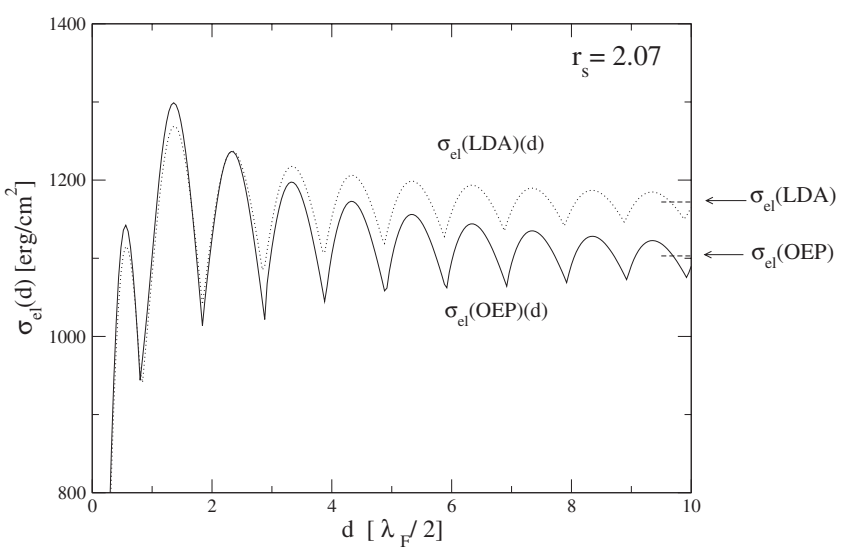

FIG. 4. Electrostatic surface energy, as a function of slab width $d$, for $r_{s}=2.07$, from Eq. (43), with $l=$ el. Full line, OEP results; dotted line, LDA results. The two arrows on the right denote the extrapolated asymptotic values $\sigma_{\mathrm{el}}(\mathrm{OEP}) \rightarrow 1103 \mathrm{erg} / \mathrm{cm}^{2}$, $\sigma_{\mathrm{el}}(\mathrm{LDA}) \rightarrow 1172 \mathrm{erg} / \mathrm{cm}^{2}$. 


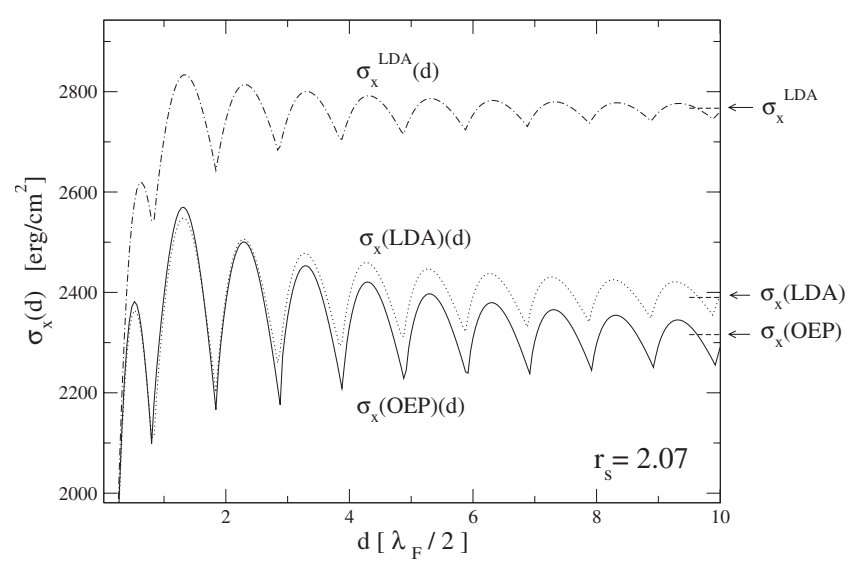

FIG. 5. Exchange surface energy, as a function of slab width $d$, for $r_{s}=2.07$, from Eq. (43), with $l=x$. Full line, OEP results; dotted line, LDA results; dash-dotted line, standard LDA-exchange results. The three arrows on the right denote the extrapolated asymptotic values $\sigma_{x}(\mathrm{OEP}) \rightarrow 2316 \mathrm{erg} / \mathrm{cm}^{2}, \sigma_{x}(\mathrm{LDA}) \rightarrow 2390 \mathrm{erg} / \mathrm{cm}^{2}$, and $\sigma_{x}^{\mathrm{LDA}} \rightarrow 2767 \mathrm{erg} / \mathrm{cm}^{2}$.

from the formally exact Eq. (41)] are obtained by using either the $x$-only self-consistent LDA eigenfunctions $\xi_{i}^{\mathrm{LDA}}(z)$ and eigenvalues $\varepsilon_{i}^{\text {LDA }}$ or their $x$-only OEP counterparts $\xi_{i}^{\mathrm{OEP}}(z)$ and $\varepsilon_{i}^{\mathrm{OEP}}$. For comparison, we have also calculated standard LDA-exchange surface energies, ${ }^{1}$

$$
\sigma_{x}^{\mathrm{LDA}}=\frac{1}{2} \int_{-\infty}^{\infty} d z n^{\mathrm{LDA}}(z)\left\{\varepsilon_{x}^{\mathrm{unif}}\left[n^{\mathrm{LDA}}(z)\right]-\varepsilon_{x}^{\mathrm{unif}}(\bar{n})\right\},
$$

where $\varepsilon_{x}^{\text {unif }}(n)$ is the exchange energy per particle of a uniform electron gas of density $n, \varepsilon_{x}^{\text {unif }}(n)=-3 e^{2}\left(3 \pi^{2} n\right)^{1 / 3} /$ $(4 \pi)$, and $n^{\mathrm{LDA}}(z)$ represents the $x$-only LDA electron density.

All $\sigma_{x}(d)(\mathrm{LDA}), \sigma_{x}(d)(\mathrm{OEP})$, and $\sigma_{x}^{\mathrm{LDA}}(d)$ exhibit the characteristic oscillatory behavior also shown by the other components of the surface energy. As in the case of the electrostatic surface energy, the onset for the filling of a new slab discrete level is associated with a minimum. Figure 5 shows that while the LDA [see Eq. (44)] considerably overestimates the exchange surface energy, which is a known result, the exact-exchange surface energy is not very sensitive to the actual shape of the single-particle orbitals and energies, i.e., to whether LDA or OEP orbitals are used. Following the extrapolation procedure of Ref. 48, we have obtained the infinite-width surface energies indicated by arrows in Fig. 5: $\sigma_{x}^{\mathrm{LDA}}=2767 \mathrm{erg} / \mathrm{cm}^{2}, \sigma_{x}(\mathrm{LDA})=2390 \mathrm{erg} / \mathrm{cm}^{2}$ (both as reported in Ref. 48), and $\sigma_{x}(\mathrm{OEP})=2316 \mathrm{erg} / \mathrm{cm}^{2}$.

We have also computed kinetic, electrostatic, and exchange surface energies for other values of the electrondensity parameter $r_{s}$, and we have obtained the infinite-width extrapolated results shown in Table I. A comparison of the LDA and OEP calculations presented in Table I shows that (i) LDA orbitals being more delocalized than the more realistic OEP orbitals, surface energies that are based on the use of LDA orbitals are too large relative to those obtained with the use of OEP orbitals, and (ii) the sum of kinetic, electrostatic, and exchange surface energies are not very sensitive to whether LDA or OEP is used in the evaluation of the singleparticle KS eigenfunctions and eigenvalues.

\section{WORK FUNCTION}

The work function $W$ is the minimum work that must be done to remove an electron from the metal at zero temperature. In the context of DFT, the rigorous expression for the work function for a slab of thickness $d$ is ${ }^{51}$

$$
W(d)=V_{\mathrm{KS}}(\infty)-\mu,
$$

where $\mu$ is the chemical potential. We note that as we are considering an electron system that is infinite in the $x-y$ plane, electronic relaxation effects after removal of one electron are infinitesimal. For a slab geometry, the work function becomes size dependent through the chemical potential $\mu(\bar{n}, d)$. We are imposing the boundary condition $V_{\mathrm{KS}}(\infty)$ $=0$; accordingly, $W(d)=-\mu>0$. Besides, the only energy of the full KS spectrum which has a physical significance is precisely the energy of the highest occupied level, which can be identified with $\mu .^{52}$ The work function for a slab with $r_{s}$ $=2.07$ and $d=4 \lambda_{F}$ is shown schematically in Fig. 1. For this particular case, nine SDLs are occupied and $\mu$ is between the ninth and tenth SDLs.

Now we focus on the slab-width dependence of the work function. Figure 6 shows the result of the $x$-only calculations that we have performed within LDA and OEP $\left[W^{\mathrm{LDA}}\right.$ and $\left.W^{\mathrm{OEP}}\right]$ for $r_{s}=2.07$. The weakly oscillating $x$-only $W^{\mathrm{LDA}}(d)$ is

TABLE I. Infinite-width extrapolated results for exchange-only kinetic $\left[\sigma_{K}\right.$ (LDA) and $\left.\sigma_{K}(\mathrm{OEP})\right]$, electrostatic [ $\sigma_{\mathrm{el}}(\mathrm{LDA})$ and $\sigma_{\mathrm{el}}$ $(\mathrm{OEP})]$, and exchange $\left[\sigma_{x}^{\mathrm{LDA}}, \sigma_{x}(\mathrm{LDA})\right.$, and $\left.\sigma_{x}(\mathrm{OEP})\right]$ surface energies for different values or $r_{s} . \sigma(\mathrm{LDA})$ and $\sigma(\mathrm{OEP})$ represent the sum of the corresponding exchange-only kinetic, electrostatic, and exchange surface energies. Empty entries in $\sigma_{\text {el }}(\mathrm{OEP})$ for the two largest $r_{s}$ studied are due to the fact that the corresponding magnitudes are so small that it is not possible to obtain a reliable extrapolated value. Units are $\mathrm{erg} / \mathrm{cm}^{2}$.

\begin{tabular}{cccccccccc}
\hline \hline$r_{s}$ & $\sigma_{K}(\mathrm{LDA})$ & $\sigma_{K}(\mathrm{OEP})$ & $\sigma_{\mathrm{el}}(\mathrm{LDA})$ & $\sigma_{\mathrm{el}}(\mathrm{OEP})$ & $\sigma_{x}^{\mathrm{LDA}}$ & $\sigma_{x}(\mathrm{LDA})$ & $\sigma_{x}(\mathrm{OEP})$ & $\sigma(\mathrm{LDA})$ & $\sigma(\mathrm{OEP})$ \\
\hline 2.00 & -5707 & -5579 & 1390 & 1317 & 3131 & 2726 & 2649 & -1591 \\
2.07 & -4832 & -4720 & 1172 & 1103 & 2767 & 2390 & 2316 & -1270 & -1613 \\
3.00 & -770 & -733 & 189 & 177 & 707 & 568 & 535 & -13 & -21 \\
4.00 & -169 & -155 & 49 & 48 & 243 & 180 & 161 & 60 & 54 \\
5.00 & -46 & -39 & 19 & & 105 & 71 & 59 & 44 \\
6.00 & -13 & -9 & 9 & & 52 & 32 & 23 & 28 \\
\hline \hline
\end{tabular}




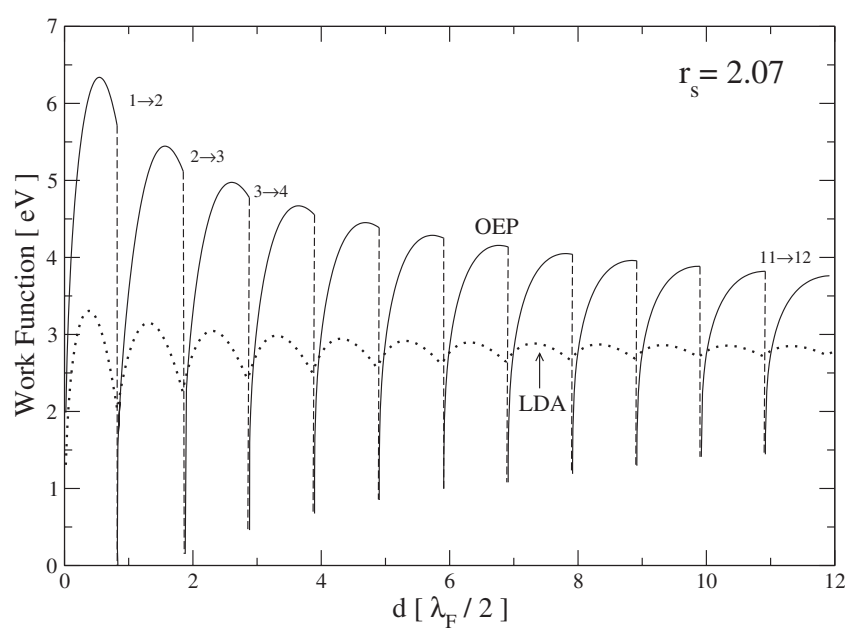

FIG. 6. Slab work function versus slab width $d$ for $r_{s}=2.07$. Full line, OEP result; dotted line, LDA result. Occupation events corresponding to transitions from a slab with $m$ occupied SDL toward $m+1$ occupied SDL are denoted as $m \rightarrow m+1$.

equivalent to the slab-width dependent work-function reported by Schulte a long-time ago. ${ }^{53}$ As discussed by Schulte, the oscillations in $W^{\mathrm{LDA}}(d)$ are the result of a combination of the shift of the bottom of the slab potential well and an effective film thickness shift, both effects suffering from an abrupt change each time the number of occupied SDL changes by one. The important point here, however, is the much stronger oscillations found in our $W^{\mathrm{OEP}}(d)$ calculations, whose explanation is provided now with some detail.

First of all, we note that, strictly speaking, the OEP work function $W^{\mathrm{OEP}}(d)$ exhibits discontinuities of large size each time a new SDL becomes infinitesimally occupied. The first discontinuity in Fig. 6 appears at the $1 \mathrm{SDL} \rightarrow 2 \mathrm{SDL}$ transition (for $d \leqslant \lambda_{F} / 2$ ), the second discontinuity appears at the $2 \mathrm{SDL} \rightarrow 3 \mathrm{SDL}$ transition (for $d \lesssim \lambda_{F}$ ), and so on. In order of clarify the source of such a discontinuous behavior, we have plotted in Fig. 7 the OEP exchange potential for slightly increasing values of the slab width $d$, around the 6 SDL $\rightarrow 7$ SDL transition. Each slab width $d$ is characterized by a "filling factor" of the last occupied SDL, which is defined as follows:

$$
\alpha_{m} \equiv \frac{\mu-\varepsilon_{m}}{\varepsilon_{m+1}-\varepsilon_{m}} .
$$

Hence, $\alpha_{m} \rightarrow 0^{+}$(implying $\mu \rightarrow \varepsilon_{m}^{+}$) corresponds to an infinitesimally small filling of the last occupied SDL $(i=m)$, while $\alpha_{m} \rightarrow 1^{-}$corresponds to the threshold of occupancy of the next SDL $(i=m+1)$. The key point here is the dramatic change in $V_{x}(z)$ when passing from the slab thickness corresponding to $\alpha_{6}=1^{-}$to the infinitesimally thicker slab corresponding to $\alpha_{7}=0^{+}\left(\sim 10^{-5}\right)$. The remaining curves have been obtained for slab widths corresponding to the seventh SDL being progressively occupied; as $\alpha_{7}$ increases from $0^{+}$to $1^{-}$, $V_{x}(z)$ approaches the form it had at $\alpha_{6}=1^{-}$, both in depth and asymptotic behavior, the only difference being a lateral shift of $V_{x}(z)$ to the right that is simply due to the larger value of $d$.

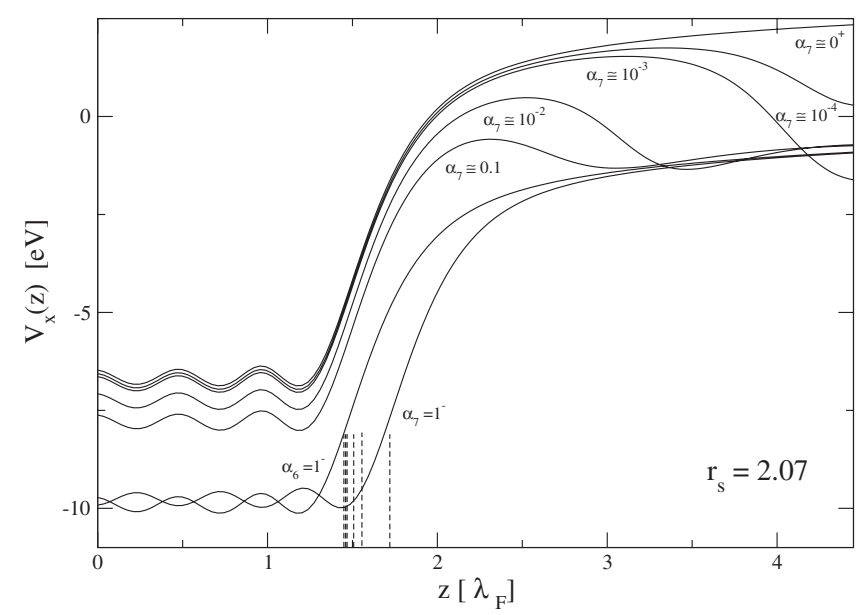

FIG. 7. Self-consistent OEP exchange potential, around the 6 $\rightarrow 7$ SDL transition, for $r_{s}=2.07$. The origin of coordinate $z$ for each slab has been taken at the slab center. The position of the right slab edge has been indicated by a vertical dashed line for each case.

Second, we note that the potential barrier that forms at the interface, right after the jellium edge on the vacuum side of the surface, exhibits both $V_{x, 1}(z)$ and $V_{x, 2}(z)$ contributions [see Eq. (20)], so the KLI approximation [which sets $\left.V_{x, 2}(z) \equiv 0\right]$ cannot be used for the analysis of the characteristic discontinuous behavior of the work function. In all cases in Fig. 7, $V_{x}(z \rightarrow \infty) \rightarrow 0$. While this is clearly seen in the figure for the curves corresponding to $\alpha_{6}=1^{-}$and $\alpha_{7}$ $=1^{-}$in which case $k_{F}^{m} \sim 1 / d$; see the asymptotics of Eq. (35)], it is not evident at all for the set of potentials with small occupancies of the last occupied level, i.e., $\alpha_{7} \ll 1$. In this case, $k_{F}^{m} \ll 1 / d$ and the asymptotic regime only takes place at $z$ coordinates that go to infinity (as $\alpha_{7} \rightarrow 0^{+}$) far beyond the $z$ coordinates considered in Fig. 7. This is the situation for $\alpha_{7} \simeq 10^{-5}, 10^{-4}$, and $10^{-3}$. As a final remark on this figure, it is important to realize that in the bulk and near the interface the exchange potentials $V_{x}(z)$ corresponding to $\alpha_{6}=1^{-}$and $\alpha_{7}=0^{+}$are simply related through a single vertical (constant) shift. This property, which can be verified numerically from Fig. 7, may also be derived analytically (see below). Finally, we note that although we have restricted our discussion to the case of a particular SDL transition, the same happens at every highest occupied $\rightarrow$ lowest unoccupied SDL transition.

With the aim of understanding how this discontinuous behavior of $V_{x}(z)$ versus the slab width explains the results of Fig. 6 for the work function $W^{\mathrm{OEP}}(d)$, we show in Fig. 8 the slab OEP electronic structure just before occupation of the SDL 7 (left panel), that is, at the slab width corresponding to $\alpha_{6} \rightarrow 1^{-}$, and just after occupation of the SDL 7 (right panel), i.e., at the slab width corresponding to $\alpha_{7} \rightarrow 0^{+}$. We note that while the Hartree potential approaches zero outside the surface exponentially and remains essentially unaffected by the infinitesimal population of the SDL 7 (compare left and right panels of Fig. 8), the OEP exchange potential [and therefore $V_{\mathrm{KS}}(z)$ as well] suffers the abrupt jump explained in Fig. 7 which induces in turn the corresponding abrupt jump in the Fermi level. The net result in going from the left to the right panels of Fig. 8 is that the work function $W^{\mathrm{OEP}}(d)$ suffers an 

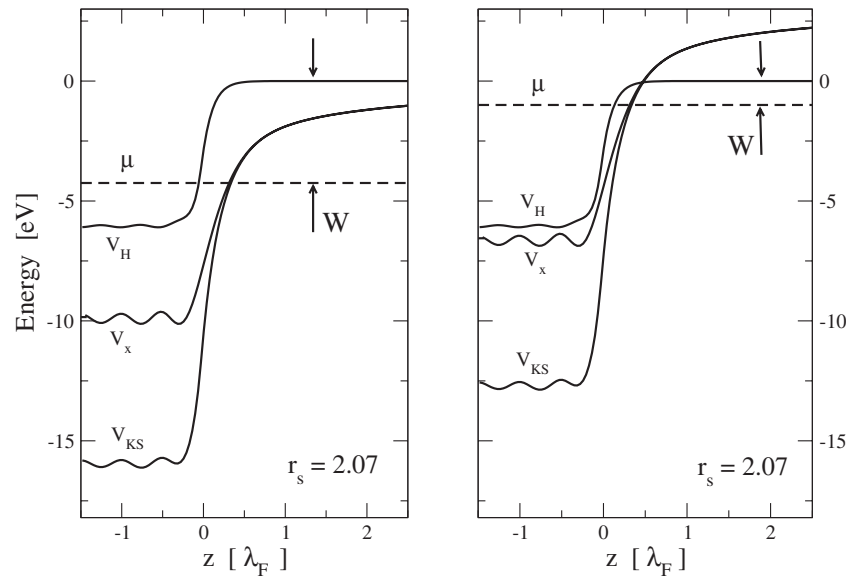

FIG. 8. Left: electronic structure of the slab for $\alpha_{6}=1^{-}$. Right: electronic structure of the slab for $\alpha_{7}=0^{+}$. The work-function $W$ jumps discontinuously from its left large value toward the smaller right value. Slab edge is at $z=0$.

abrupt (discontinuous) decrease, as the boundary condition $V_{\mathrm{KS}}(\infty)=0$ is rigorously valid in both cases. This discontinuous behavior of $W^{\mathrm{OEP}}(d)$, shown schematically in Fig. 8, represents precisely the origin of the jumps that are visible in Fig. 6 at every threshold for SDL occupation. It is evident from Fig. 6 that the size of the discontinuity decreases as $d$ increases.

Finally, we investigate the size of the discontinuities that are visible in Fig. 6. For this, we rewrite the central OEP equation [as given by Eq. (14)] in the following way:

$$
\begin{aligned}
& \sum_{i=1}^{m-1}\left(k_{F}^{i}\right)^{2} \int_{-\infty}^{\infty}\left[V_{x}\left(z^{\prime} ; m\right)-u_{x}^{i}\left(z^{\prime} ; m\right)\right] G_{i}^{\mathrm{KS}}\left(z, z^{\prime}\right) \varphi_{i}\left(z^{\prime}, z\right) d z^{\prime} \\
& \quad+\left(k_{F}^{m}\right)^{2} \int_{-\infty}^{\infty}\left[V_{x}\left(z^{\prime} ; m\right)-u_{x}^{i}\left(z^{\prime} ; m\right)\right] G_{m}^{\mathrm{KS}}\left(z, z^{\prime}\right) \varphi_{m}\left(z^{\prime}, z\right) d z^{\prime} \\
& \quad+\text { c.c. }=0
\end{aligned}
$$

where $\varphi_{i}\left(z, z^{\prime}\right)=\xi_{i}(z)^{*} \xi_{i}\left(z^{\prime}\right)$. In writing Eq. (47) the contribution of all the $m-1$ occupied SDLs has been split from the contribution of the last occupied $(m)$ SDL. The label $m$ in $V_{x}(z ; m)$ and $u_{x}^{i}(z ; m)$ has been introduced in order to emphasize that they are solutions of a system with $m$ occupied SDLs.

Let us now define a distance $Z$, such that for $z>Z$ the electron density is dominated by the contribution of the last occupied $(m)$ SDL, which is the one with the slowest decay. Equation (6) clearly shows that $Z \rightarrow \infty$ when $k_{F}^{m} \rightarrow 0$, which is the case whenever $\alpha_{m} \rightarrow 0^{+}$, i.e., whenever the filling of the last occupied SDL is infinitesimally small. We consider the following trial solution of Eq. (47):

$$
V_{x}(z ; m)=V_{x}(z ; m-1)+C_{x}(m)
$$

for $z<Z$ and $k_{F}^{m} \rightarrow 0$, with $C_{x}(m)$ being a constant which depends on the last occupied SDL. Introducing this trial solution into Eq. (47), we obtain

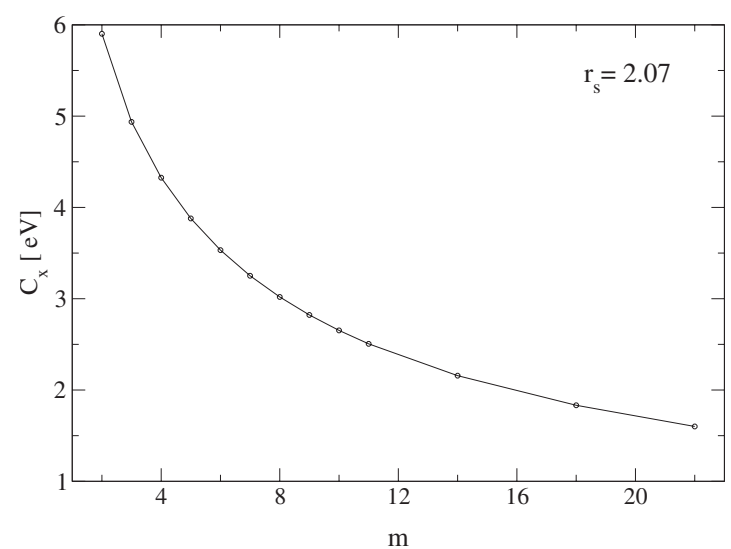

FIG. 9. Exchange-driven discontinuity $C_{x}(m)$ for increasing number of occupied slab levels, as follows from Eq. (51).

$$
\begin{aligned}
& \sum_{i=1}^{m-1}\left(k_{F}^{i}\right)^{2} \int_{-\infty}^{\infty}\left[V_{x}\left(z^{\prime} ; m-1\right)+C_{x}(m)\right. \\
& \left.\quad-u_{x}^{i}\left(z^{\prime} ; m\right)\right] G_{i}^{\mathrm{KS}}\left(z, z^{\prime}\right) \varphi_{i}\left(z^{\prime}, z\right) d z^{\prime}+\left(k_{F}^{m}\right)^{2} \\
& \quad \times \int_{-\infty}^{\infty}\left[V_{x}\left(z^{\prime} ; m-1\right)+C_{x}(m)\right. \\
& \left.\quad-u_{x}^{i}\left(z^{\prime} ; m\right)\right] G_{m}^{\mathrm{KS}}\left(z, z^{\prime}\right) \varphi_{m}\left(z^{\prime}, z\right) d z^{\prime}+\text { c.c. }=0 .
\end{aligned}
$$

In the limit $k_{F}^{m} \rightarrow 0$, the second-line contribution of Eq. (49) is arbitrarily small; also, the KS wave functions $\xi_{i}(z)$ and eigenvalue differences (denominators) entering $G_{i}^{\mathrm{KS}}\left(z, z^{\prime}\right)$ should be extremely similar for the slab width corresponding to $m-1$ occupied levels and $\alpha_{m-1} \rightarrow 1^{-}$and the slab width corresponding to $m$ occupied levels and $\alpha_{m} \rightarrow 0^{+}$. Therefore, an inspection of Eq. (22) leads us, using similar arguments, to the conclusion that $u_{x}^{i}(z ; m) \rightarrow u_{x}^{i}(z ; m-1)$ for all $i<m, z$ $<Z$, and $k_{F}^{m} \rightarrow 0$. Under these conditions, the first line of Eq. (49) reverts to the OEP equation for a slab width corresponding to $m-1$ occupied states, and the proposal of Eq. (48) is proved. Considering now that $C_{x}(m)=V_{x}(z ; m)-V_{x}(z ; m-1)$ and taking the expectation value at the last occupied state $(m-1)$ of $m-1$ system, we find

$$
C_{x}(m)=\bar{V}_{x}^{m-1}(m)-\bar{V}_{x}^{m-1}(m-1) .
$$

Now, for the $m-1$ system we can use the boundary condition $\bar{V}_{x}^{m-1}(m-1)=\bar{u}_{x}^{m-1}(m-1)$ and, once again, approximate $\bar{u}_{x}^{m-1}(m-1) \simeq \bar{u}_{x}^{m-1}(m)$, yielding

$$
C_{x}(m)=\bar{V}_{x}^{m-1}(m)-\bar{u}_{x}^{m-1}(m),
$$

which has the nice feature that both the exchange potential $V_{x}$ and the orbital-dependent exchange potential $u_{x}$ are referred to the $m$ system. For the $m$ system $\bar{V}_{x}^{m}(m)=\bar{u}_{x}^{m}(m)$, which does not prevent the constant $C_{x}(m)$ from being nonzero (as shown in Fig. 7) since the KS orbitals $\xi_{m-1}(z)$ and $\xi_{m}(z)$ are different. As the slab width increases, $m$ also increases and the difference between $\xi_{m-1}(z)$ and $\xi_{m}(z)$ decreases, thereby leading to the expectation that $C_{x}(m) \rightarrow 0$ as $d \rightarrow \infty$. This is explicitly shown in Fig. 9. While this analysis explains why $C_{x}(m) \neq 0$ for any finite $m$, it does not give a 
TABLE II. Infinite-width extrapolated $x$-only LDA and OEP work functions for various values of $r_{s}$. Units are $\mathrm{eV}$.

\begin{tabular}{lllllll}
\hline \hline$r_{s}$ & 2.00 & 2.07 & 3.00 & 4.00 & 5.00 & 6.00 \\
\hline$W^{\mathrm{LDA}}$ & 2.82 & 2.80 & 2.50 & 2.15 & 1.86 & 1.62 \\
$W^{\mathrm{OEP}}$ & 2.64 & 2.63 & 2.49 & 2.11 & 1.84 & 1.61 \\
\hline \hline
\end{tabular}

hint about its sign; Fig. 9 shows, however, that $C_{x}(m)$ is positive for all $m$. This positive jump in $V_{x}(z)$ is exchange driven: at each threshold width for the occupation of a new level, a barrier appears against the occupancy of an empty SDL. This is due to the fact that intra-SDL exchange is stronger than inter-SDL exchange. As a consequence, the slab gains exchange energy by restricting new SDL occupancies. On the other hand, correlation induces in general a negative jump in $V_{c}(z)$, so the net jump in $V_{\mathrm{xc}}(z)$ depends on the relative weight of exchange and correlation for each particular system. ${ }^{54}$

Finally, we have observed numerically that the average of the OEP work functions for slab widths corresponding to $\alpha_{m-1} \rightarrow 1^{-}$and $\alpha_{m} \rightarrow 0^{+}$remains the same (within error bars) for all the $m$ values that we have considered. Hence, we have taken the infinite-width extrapolated work function to be simply that average. Table II exhibits the infinite-width $x$-only LDA and OEP work functions that we have obtained in this way for various values of the electron-density parameter $r_{s}$. OEP work functions are slightly and systematically smaller than their LDA counterparts.

\section{CONCLUSIONS}

We have reported benchmark exact-exchange selfconsistent calculations of the KS potential, surface energy, and work function of jellium slabs in the framework of the OEP scheme. Special emphasis has been put into the asymptotical behavior of the exact-exchange KS potential far into the vacuum and the large quantum size effects that are present in the slab-width dependence of the surface energy and work function.

We have performed a detailed analysis of the asymptotics of the exact-exchange KS potential far into the vacuum, ${ }^{39}$ showing that at a distance $z$ that is larger than the slab thickness the exact-exchange potential takes an imagelike form, $V_{x}(z \rightarrow \infty) \rightarrow-e^{2} / z$, but with a coefficient that differs from that of the classical image potential $V_{\mathrm{im}}(z)=-e^{2} / 4 z$. Although this result has been obtained in the $x$-only approximation, it is also true in the presence of correlation due to the separability of the basic OEP equations in their basic exchange and correlation components.

The OEP kinetic, electrostatic, and exchange contributions to the surface energy of jellium slabs have been obtained as a function of the slab width $d$ and for a set of electron densities characterized by the parameter $r_{s}$. We have shown that these components of the surface energy are all oscillating functions of $d$, with the oscillating period being $\approx \lambda_{F} / 2$. By a suitable extrapolation procedure, we have found the values of the different components of the surface energy of a semi-infinite jellium. We have compared our OEP surface energies with those obtained from the same formally exact expressions [see Eqs. (39)-(41)] but using single-particle LDA wave functions and energies; we have found small differences between these OEP and LDA surface energies, which appear as a consequence of the LDA orbitals being slightly more delocalized (diffuse) than their more realistic OEP counterparts.

Finally, we have performed $x$-only OEP calculations of the work function of jellium slabs, again as a function of the slab width $d$. We have found that the OEP work-function exhibits large quantum size effects that are absent in the LDA and which reflect the intrinsic derivative discontinuity of the exact KS potential. The amplitude of this discontinuity diminishes as the slab width increases and becomes arbitrarily small as $d \rightarrow \infty$, i.e., in the case of a semi-infinite system. This has been proved both analytically and numerically. We also note that although the precise value of the $x$-only OEP work functions reported here would change with the inclusion of correlation, the exact slab work function is expected to exhibit the large quantum size effects and discontinuities observed in the present work, barring possible accidental cancellations of exchange-driven and correlationdriven contributions to the total discontinuity. The presence of these large discontinuities in the $x$-only OEP slab work function (and presumably also in the actual work function that includes correlation) highlights the potential danger in which can be incurred by performing elaborated calculations for a restricted set of slab sizes without performing a suitable and reliable extrapolation toward the semi-infinite case. In summary, we expect that the benchmark exact-exchange OEP calculations reported here for jellium slabs will serve as motivation and as a starting point for the development of more realistic approximations for the exchange-correlation energy functional of jellium and real surfaces.

\section{ACKNOWLEDGMENTS}

C.M.H. wishes to acknowledge the financial support received from CONICET of Argentina. J.M.P. acknowledges partial support by the University of the Basque Country, the Basque Unibertsitate eta Ikerketa Saila, the Spanish Ministerio de Educación y Ciencia (Grants No. FIS2006-01343 and No. CSD2006-53), and the EC Sixth Framework Network of Excellence NANOQUANTA (Grant No. NMP4-CT-2004500198). C.R.P. was supported by the European Community through a Marie Curie IIF (Grant No. MIF1-CT-2006040222). 
*Permanent address: Centro Atómico Bariloche and Instituto Balseiro, 8400 S. C. de Bariloche, Río Negro, Argentina.

${ }^{1}$ N. D. Lang and W. Kohn, Phys. Rev. B 1, 4555 (1970).

${ }^{2}$ N. D. Lang, in Solid State Physics, edited by H. Eirenreich, F. Seitz, and D. Turnball (Academic, New York, 1973), Vol. 28, p. 225.

${ }^{3}$ R. G. Parr and W. Yang, Density Functional Theory of Atoms and Molecules (Oxford University Press, New York, 1989).

${ }^{4}$ R. M. Dreizler and E. K. U. Gross, Density Functional Theory: An Approach to the Quantum Many-Body Problem (SpringerVerlag, Heidelberg, 1990).

${ }^{5}$ W. Kohn and L. J. Sham, Phys. Rev. 140, A1133 (1965).

${ }^{6}$ M. Nekovee and J. M. Pitarke, Comput. Phys. Commun. 137, $123(2001)$

${ }^{7}$ Z. Y. Zhang, D. C. Langreth, and J. P. Perdew, Phys. Rev. B 41, 5674 (1990)

${ }^{8}$ L. A. Constantin, J. P. Perdew, and J. Tao, Phys. Rev. B 73, 205104 (2006).

${ }^{9}$ E. Krotscheck, W. Kohn, and G.-X. Qian, Phys. Rev. B 32, 5693 (1985).

${ }^{10}$ E. Krotscheck and W. Kohn, Phys. Rev. Lett. 57, 862 (1986).

${ }^{11}$ A. G. Eguiluz, M. Heinrichsmeier, A. Fleszar, and W. Hanke, Phys. Rev. Lett. 68, 1359 (1992).

${ }^{12}$ X. P. Li, R. J. Needs, R. M. Martin, and D. M. Ceperley, Phys. Rev. B 45, 6124 (1992).

${ }^{13}$ P. H. Acioli and D. M. Ceperley, Phys. Rev. B 54, 17199 (1996).

${ }^{14}$ B. Wood, N. D. M. Hine, W. M. C. Foulkes, and P. GarcíaGonzález, Phys. Rev. B 76, 035403 (2007).

${ }^{15}$ L. A. Constantin, J. M. Pitarke, J. F. Dobson, A. García-Lekue, and J. P. Perdew, Phys. Rev. Lett. 100, 036401 (2008).

${ }^{16}$ T. Grabo, J. Kreibich, S. Kurth, and E. K. U. Gross, in Strong Coulomb Interactions in Electronic Structure Calculations: Beyond the Local Density Approximation, edited by V. I. Anisimov (Gordon and Breach, Amsterdam, 2000).

${ }^{17}$ E. Engel, in A Primer in Density Functional Theory, Lecture Notes in Physics Vol. 620, edited by C. Fiolhais, F. Nogueira, and M. A. L. Marques (Springer, Berlin, 2003), p. 56.

${ }^{18}$ J. D. Talman and W. F. Shadwick, Phys. Rev. A 14, 36 (1976).

${ }^{19}$ J. P. Perdew and A. Zunger, Phys. Rev. B 23, 5048 (1981).

${ }^{20}$ F. Della Sala and A. Görling, Phys. Rev. Lett. 89, 033003 (2002); J. Chem. Phys. 116, 5374 (2002).

${ }^{21}$ D. M. Bylander and L. Kleinman, Phys. Rev. Lett. 74, 3660 (1995); M. Städele, J. A. Majewski, P. Vogl, and A. Görling, ibid. 79, 2089 (1997); Y.-H. Kim and A. Gorling, ibid. 89, 096402 (2002).

${ }^{22}$ S. J. A. van Gisbergen, P. R. T. Schipper, O. V. Gritsenko, E. J. Baerends, J. G. Snijders, B. Champagne, and B. Kirtman, Phys. Rev. Lett. 83, 694 (1999).

${ }^{23}$ T. Kreibich, S. Kurth, T. Grabo, and E. K. U. Gross, Adv. Quantum Chem. 33, 31 (1998).

${ }^{24}$ J. B. Krieger, Y. Li, and G. J. Iafrate, Phys. Rev. A 45, 101 (1992).

${ }^{25}$ J. P. Perdew, R. G. Parr, M. Levy, and J. L. Balduz, Phys. Rev. Lett. 49, 1691 (1982).

${ }^{26}$ J. P. Perdew and M. Levy, Phys. Rev. Lett. 51, 1884 (1983).

${ }^{27}$ J. P. Perdew, NATO ASI Ser., Ser. B 123, 265 (1985).

${ }^{28}$ D. J. Tozer and N. C. Handy, J. Chem. Phys. 108, 2545 (1998).

${ }^{29}$ Y.-H. Kim, I.-H. Lee, S. Nagaraja, J. P. Leburton, R. Q. Hood, and R. M. Martin, Phys. Rev. B 61, 5202 (2000); P. GarcíaGonzález, ibid. 62, 2321 (2000); L. Pollack and J. P. Perdew, J.
Phys.: Condens. Matter 12, 1239 (2000); P. García-González and R. W. Godby, Phys. Rev. Lett. 88, 056406 (2002).

${ }^{30}$ The electron-density parameter $r_{s}$ is defined as the radius of a sphere containing on average one electron, i.e., $r_{s}$ $=\left(3 / 4 \pi \bar{n} a_{0}^{3}\right)^{1 / 3}$. A convenient length unit for the present system is $\lambda_{F}=\left(32 \pi^{2} / 9\right)^{1 / 3} r_{s} a_{0} \approx 3.274 r_{s} a_{0}$.

${ }^{31} \mathrm{We}$ have found that two infinite barriers located at $2 \lambda_{F}$ from each jellium edge are enough for this purpose in our system.

${ }^{32}$ Note that we have included in our Hartree potential the contribution which comes from the uniform jellium background (proportional to $n_{+}$). Alternatively, this contribution may be denoted separately as the "external potential."

${ }^{33}$ Note that dimensions of Eq. (6) are (length) ${ }^{-3}$, as correspond to our three-dimensional (3D) system. However, for the particular slab geometry, the number density only depends on one spatial coordinate $(z)$.

${ }^{34}$ Due to the imposed translational invariance in the $x, y$ plane, functional derivatives in this work are conveniently defined as $\delta f=\int[\delta f[g] / \delta g(z)] \delta g(z) d z$, where $\delta g(z)$ represents a uniform variation of the function $g(\mathbf{r})$ in the plane $\mathbf{r}=z$. This is the origin of the factor $A^{-1}$ in Eq. (7)

${ }^{35} \mathrm{We}$ assume that the xc energy functional does not depend on the unoccupied eigenfunctions and eigenvalues of the KS equation, which is only known to be true in the case of the $x$-only energy functional. In general, the sum over the index $i$ should run over the whole KS spectrum (Refs. 54 and 55). Also, for simplicity, we do not consider the possibility that $E_{\mathrm{xc}}$ depends on the KohnSham eigenvalues. Once again, this is only known to be true in the case of the $x$-only energy functional for fixed particle number. This is however, the only case to which all the numerical calculations presented below apply.

${ }^{36}$ In the $x$-only version it can be written as Eq. (22).

${ }^{37}$ S. Kummel and J. P. Perdew, Phys. Rev. Lett. 90, 043004 (2003); Phys. Rev. B 68, 035103 (2003).

${ }^{38}$ S. Rigamonti, C. R. Proetto, and F. A. Reboredo, Europhys. Lett. 70, 116 (2005).

${ }^{39}$ C. M. Horowitz, C. R. Proetto, and S. Rigamonti, Phys. Rev. Lett. 97, 026802 (2006). Note that there is a sign of difference between the definition of the shifts in this and the present work.

${ }^{40}$ Note that the equivalence is only valid for this specific case (shifts identically zero). As soon as the shifts are different from zero, $V_{\mathrm{xc}, 1}(z)$ and $V_{\mathrm{xc}}^{\mathrm{KLI}}(z)$ will be not equal due to the presence of $V_{\mathrm{xc}, 2}(z)$ in this more general situation.

${ }^{41}$ W. Kohn and A. E. Mattsson, Phys. Rev. Lett. 81, 3487 (1998).

${ }^{42}$ M. Abramowitz and I. A. Stegun, Handbook of Mathematical Functions (Dover, New York, 1964).

${ }^{43}$ J. Jung, J. E. Alvarellos, E. Chacon, and P. Garcia-Gonzalez, J. Phys.: Condens. Matter 19, 266008 (2007).

${ }^{44}$ The first approximation $k_{F}^{m}\left|z-z^{\prime}\right| \simeq k_{F}^{m} z$ can be justified for $z$ $\rightarrow \infty$, even considering that the $z^{\prime}$ integral covers the range from $-\infty$ to $+\infty$. This is due to the fact that the main contribution to this integral comes from values of $z^{\prime}$ inside the slab, so the range of $z^{\prime}$ could be effectively restricted to the region $-d \lesssim z^{\prime} \lesssim 0$.

${ }^{45}$ Note that $\bar{z}^{m}=-d / 2$. So $\beta$ is always negative as a sum of two negative terms.

${ }^{46}$ Fred Nastos, Ph.D. thesis, Queen's University, 2000.

${ }^{47}$ C. M. Horowitz, C. R. Proetto, and J. M. Pitarke (unpublished).

${ }^{48}$ J. M. Pitarke and A. G. Eguiluz, Phys. Rev. B 57, 6329 (1998); 63, 045116 (2001).

${ }^{49}$ We have taken then $E_{K}^{\text {unif }}(d)=e^{2}\left(3 \pi^{2} n\right)^{5 / 3} d /\left(20 \pi^{2} a_{0}\right), E_{\mathrm{el}}^{\text {unif }}(d)$ $=0$, and $E_{x}^{\text {unif }}(d)=-3 e^{2}\left(3 \pi^{2}\right)^{1 / 3}(n)^{4 / 3} d /\left(4 \pi a_{0}\right)$, where $n$ is the density of the uniform electron gas. 
${ }^{50}$ By $x$-only LDA we mean that in the KS equations the actual exchange potential $V_{x}(z)$ is replaced at this point by the exchange potential of a uniform electron gas with the local density, i.e., $V_{x}^{\mathrm{LDA}}(z)=-[6 n(z) / \pi]^{1 / 3}$.

${ }^{51}$ See Ref. 7. Note that as the work function is defined as the difference between two energies, it is independent of the choice for the zero energy factor (ef) energy.
${ }^{52}$ F. K. Schulte, J. Phys. C 7, L370 (1974).

${ }^{53}$ F. K. Schulte, Surf. Sci. 55, 427 (1976).

${ }^{54}$ S. Rigamonti and C. R. Proetto, Phys. Rev. Lett. 98, 066806 (2007).

${ }^{55}$ S. Rigamonti and C. R. Proetto, Phys. Rev. B 73, 235319 (2006). 